\title{
Yoga as A Complementary Therapeutic Modality for People with Alzheimer's Disease: A Review
}

\author{
Max Ghaffari* \\ Department of Nursing, Benedictine University, USA
}

Submission: November 11, 2019; Published: December 04, 2019

*Corresponding author: Max Ghaffari, Department of Nursing, Benedictine University, Chicago, USA

\begin{abstract}
Yoga is used by many to promote general wellness and disease prevention. It is also increasingly used as a complementary treatment for symptoms of various medical conditions, including depression, anxiety, low back pain, musculoskeletal conditions, asthma, hypertension, and cancer. In addition to these benefits, yoga also impacts cognitive functioning. Yoga therapy is a new and emerging field, and its clinical therapeutic use have yet to be truly defined. The research on the effectiveness of medical yoga for people who have Alzheimer's disease is still in its early stages, but as shown in this review, it continues to expand and advance. The aim of this Review is to summarize the findings from completed clinical trials that have tested the effectiveness of yoga therapy alone or in combination with other related interventions in people with Alzheimer's disease.
\end{abstract}

Keywords: Yoga therapy; Medical yoga; Alzheimer's disease; Yoga and Alzheimer's disease; Yoga and cognitive functioning; Yoga and depression; Yoga and CAM; Dementia and CAM

Abbrevations: AD: Alzheimer's Disease; MMSE: Mini Mental State Examination; NCCIH: National Center for Complementary and Integrative Health; RCTs: Review of Published Randomized Controlled Trials

\section{Introduction}

Increased age is related to an increased probability of exhibiting cognitive deficits related to Alzheimer's disease (AD). The progressive ageing of the global population is leading to an increased prevalence of age-related disorders; such as Alzheimer's disease (AD). During the last few years; a growing number of studies has provided evidence for cognitive improvements during healthy and pathological ageing after applying non-pharmacological interventions. Dementia is a clinical syndrome characterized by cognitive decline; motor deficits and/or behavioral problems; causing a decline in daily functioning. Various neuro pathologies can underlie dementia syndromes, but the most prevalent cause is Alzheimer's disease (AD). This accounts for $60-70 \%$ of the cases [1]. As of 2013; there were an estimated 44.4 million people worldwide with dementia. This number will increase to an estimated 75.6 million in 2030 and 135.5 million in 2050 [2].

Dementia refers to a loss of cognitive function; resulting in decreased understanding; perception; or awareness of one's thoughts and ideas. Some risk factors for dementia include age; atherosclerosis; genetics; head trauma; and various infections of the brain and body; such as syphilis or human immunodeficiency virus [1]. Dementia may occur gradually or quickly and can be caused by brain changes associated with disease or trauma. An estimated 2 million Americans suffer from severe dementia; 1 to 5 million experience mild to moderate dementia. Some causes of dementia are preventable; however; many causes of dementia; such as Alzheimer disease; have no cure. Pharmaceutical treatments only slow symptom progression. Integrative therapeutics may offer complementary options to patients interested in such modalities [1].

\section{Literature Review}

Initially; relevant texts were identified by general internet searches using Google Scholar. Then; a literature search was undertaken using CINHAL; PubMed; the Cochrane database; ProQuest Central; and references of retrieved articles. The search was restricted to full text publications and those published only in English. The main search terms were yoga therapy; medical yoga; Alzheimer's disease; (yoga and Alzheimer's disease); (yoga and cognitive functioning); (yoga and depression); (yoga and CAM); (dementia and CAM). The main reason for exclusion was non-research-based articles. The author scanned all potentially relevant articles published since 1995 to examine the literature on medical yoga and its effects on AD. 


\section{Alzheimer's Disease}

In the early stages; $\mathrm{AD}$ manifests as an amnesic syndrome; which subsequently extends to other cognitive domains; such as language; visuospatial attention and executive functions. Beta-amyloid deposits and intracellular neurofibrillary tangles constitute the pathophysiological hallmarks of this neurodegenerative disease [3]. Patients with AD exhibit reduced cortical volumes in the frontal; temporal and parietal cortices. At the functional level; AD is mainly characterized by neural hyperexcitability; which correlates with the degree of brain atrophy and impaired learning ability. Likewise; studies have reported a loss of age-related compensatory activation; which was related to reduced brain plasticity mechanisms [3]. AD first results in a loss of a specific neuronal subtype (the cholinergic neurons) by reducing the expression levels of choline acetyltransferase. This enzyme breaks down acetylcholine; a neurotransmitter expressed in the neuromuscular junction; resulting in the loss of normal brain function. This loss of acetylcholine in AD patients was first noted in the late 1970s and is now known to correlate with reduced cortical choline acetyltransferase; the enzyme responsible for synthesizing acetylcholine from choline; which in turn correlates with neurotic plaque numbers and reduced MiniMental State Exam scores [3]. Nevertheless; structural damage in $\mathrm{AD}$ is not always accompanied by an equivalent degree of cognitive impairment. Different clinical severities are frequently reported for similar degrees of neural damage. These findings suggest that AD patients show differences in their ability to deploy brain compensatory mechanisms; which might be related to individual differences in residual brain plasticity [3].

It is recognized; now; that late-onset $\mathrm{AD}$ begins decades before a diagnosis of dementia; with a long prodromal phase often beginning in midlife. The earliest part of this prodromal phase is called preclinical $\mathrm{AD}$ and involves no observable cognitive symptoms but offers a large window of opportunity for early intervention [4]. Alzheimer's disease (AD) is an agerelated clinical syndrome which causes a progressive dementia in the elderly. It is characterized by a slow; hidden onset; and there must be evidence of decline from normal functioning for a clinical diagnosis to be made. The progression of the condition is divided into three stages of mild; moderate or severe; as determined by results of global cognitive test scores. The relationship between cognitive symptoms and the multiple observed pathologies in $\mathrm{AD}$ is not yet fully understood. For instance; the presence of amyloidplaques first used to identify $\mathrm{AD}$ as a distinct disorder; does not clearly correlate with AD symptoms and they can be found in healthy elderly with no cognitive impairment. The number of neurofibrillary tangles has been found to correlate with cognitive status; but this is not specific to AD [4]. Clinical cognitive tests have been validated by use in multiple dementia and healthy populations; the Mini Mental State Examination (MMSE) for instance has been shown to decline by an average of four points in one year in non-treated AD patients [4].
Currently; no disease modifying drugs for dementia are available and pharmacological treatment is limited to therapies that alleviate the symptoms. However; these treatments are not efficacious in all patients and may introduce undesirable side-effects. Current treatments focus on reducing associated symptoms; including cognitive impairment; behavioral disturbances; and mood disturbances. Non-pharmacological interventions; such as physical activity interventions; are therefore appealing alternatives or add-ons. Epidemiological studies have shown that increased lifetime engagement in physical activities reduces the risk of dementia-onset in cognitively normal elderly persons [5]. Unfortunately; pharmacological agents temporarily treat symptoms without having an effect on the underlying pathophysiology of the disease [6].

\section{Complementary and Alternative Medicine}

By definition; complementary and alternative medicine "is a group of diverse medical and health care systems; practices; and products that are not presently considered part of conventional medicine." Some of these therapies provide promise as novel and effective therapies for treatment and prevention of geriatric psychiatric disorders with generally more modest sideeffect profiles. "Complementary" generally refers to using a nonmainstream approach together with conventional Western medicine. "Alternative" refers to using a nonmainstream approach in place of conventional Western medicine. The U.S. National Center for Complementary and Integrative Health (NCCIH) of the National Institutes of Health has defined complementary health care as "health care approaches developed outside of mainstream Western; or conventional; medicine" when they are used either together with conventional medicine; or as alternative treatment in place of conventional medicine.

"Integrative medicine" is another term often used when strategy combines alternative and complementary medicine with evidence-based Western medicine. Such therapies are referred to as complementary; alternative; and integrative medicine (CAM/ CAIM). CAIM interventions have varying levels of efficacy and evidence; they can include mind-body practices; conventional physical activity; dietary interventions; and natural products; in addition to other medical system practices. Mind-body practices refer to practices whereby there is both physical and mental activity that may be combined during training. These activities can be more exercise-focused (i.e.; yoga; tai chi; qigong) or more focused on practice and stress reduction (i.e.; mindfulness and relaxation). Physical activity refers to any bodily movement produced by skeletal muscles that requires energy expenditure; and may include aerobic; resistance; stretching and toning; or combination activities. Eyre \& Lavretsky [6] posit several major reasons why practitioners need to recognize the importance of these interventions:

a) CAIM therapy use is high and rising. Research suggests 12-month prevalence of any CAIM usage (excluding prayer) in 
the United States is around 35\% to 50\%; Baby Boomers (adults born from 1946 to 1964) report significantly higher rates of use than the Silent Generation (born from 1925 to 1945) for chronic conditions.

b) The global population is aging. See earlier discussion.

c) CAIM therapies are increasingly cited in clinical guidelines. It is now

d) commonplace to note the use of therapies such as conventional exercise in the management of psychiatric conditions.

e) CAIM therapies are a source for innovative interventions and have a growing; quality empirical research basis. Empirical research into these therapies is exploring their clinical efficacy in trials and the neurobiological mechanism underlying their effects and

f) CAIM therapies can help to lower utilization of conventional medicines [6].

Complementary and alternative medicine is based on multiple healing traditions practiced long before conventional Western medicine. Emerging from diverse cultural traditions worldwide; these approaches to health and healing offer the wisdom of their unique perspective on the human condition. Although more evidence is needed on many fronts; evidencebased complementary therapies are increasingly being integrated into medical practice. When used appropriately; these approaches may expand the tools available to health care practitioners and the options that can be recommended to patients. Many patients may appreciate choices other than drugs in different conditions.

\section{Medical Yoga}

Yoga is an ancient Indian; non-religious mind-body approach classified as a form of CAM by the National Institutes of Health. Excavations in the Indus basin; made in the twentieth century uncovered intact ceramics; about five thousand years old; on which are depicted some of the postures of Yoga [7]. The word "Yoga" may be construed as referring to the union of body and mind in harmonious health and efficiency. At its highest level it refers to the unitary consciousness of the mystic; Hindu or otherwise; in which the ego is transcended and one's essential spiritual nature is realized. The "union" which has been the goal of Yoga for thousands of years aim to achieve great inner stillness [8].

Yoga consists of the practice of specific posture; regulated breathing and meditation. It is based on a harmonizing system for the body; mind; and spirit to attain inner peace and liberation. There are various review articles reporting the effect of mindbody medicine in various neurological disorders. Yoga does emphasize appropriate postural alignment; musculoskeletal strength and endurance as well as balance. Yoga; considered to be "science of the mind" as stated in the Yoga Sutras of Patanjali; is a practice of physical postures (Asanas); meditation (Dhyana); and breath (Pranayama). Yoga-based practices of postures and movement sequences are usually taught in conjunction with some type of breathing and/or meditation technique. This type of mindful movement with slow; rhythmic breathing is more likely to promote parasympathetic and vagal tone compared to other forms of exercise. Yoga practices can increase multiple neurotransmitters and hormones such as GABA; serotonin; and dopamine. They have been shown to increase levels of melatonin; helping to initiate sleep; improving sleep quality and sleep regulation; as well as increasing levels of oxytocin; the "bonding hormone"; thus, helping with feelings of connectedness and "being seen and heard" [9]. Medical yoga is defined as the use of yoga practices for the prevention and potential treatment of medical conditions.

Beyond the physical elements of yoga; which are important and effective for strengthening the body; medical yoga also incorporates appropriate breathing techniques; mindfulness; meditation and self-reflection in order to achieve the maximum benefits. Medical Yoga Therapy or "Yoga Chikitsa" is the dynamic state of physical and mental ease; coupled with spiritual wellbeing. Yoga is being adopted in conventional physical therapy practice as an integral therapy. Approximately 55\% of physical therapists regularly use yoga as a common form of alternative strength training; which may reflect its adaptability for addressing various musculoskeletal problems [9].

Yoga is identified as a complementary health approach by the National Center for Complementary and Integrative Health. Hatha yoga; the most common branch of yoga practiced in the US; emphasizes physical postures; and usually incorporates breathing and meditation. It includes some of the most well-known styles of yoga practiced in the U.S. (e.g.; Anusara; Ashtanga; Bikram; Integral; Iyengar; Kripalu) [10]. This ancient practice is very effective in improving one's well-being and emotional regulation; as well as providing stress relief and enhancing self-care. Mastering self-care and self-regulation are essential tools against mental illness; substance abuse; and advocates for a healthy body and mind. The practice of yoga and mindfulness can help to increase positive emotions; while decreasing negative feelings of depression and anxiety. Medical yoga therapy is an individualized; personalized approach to the patient; and may be integrated with any current therapy or medical regimen [9]. A systematic review of yoga research by Park and McCaffrey noted a lack of standardization of yoga programs as a deficit in fully explicating evidence of the potential benefits of yoga [10].

\section{Evidence of Effectiveness}

Depression symptoms are among the early changes in the preclinical stages of dementia syndromes. There is a significant link between worsening depression symptoms and declining cognition over two to seven years that was influenced by Alzheimer's disease pathology; as measured by PET imaging of brain amyloid 
[11]. The most recent data on geriatric depression identified depressive disorders as a leading cause of burden internationally. Prior review of published randomized controlled trials (RCTs) of yoga for depression in adults revealed that although all trials found benefit; trial methodologies have generally been weak with lack of blinding; short duration of the intervention; variable outcome measures; and limited information about subjects; randomization procedures; compliance; and dropout rates. Comparative studies of yoga have likewise been limited; with one trial demonstrating that yoga was as effective as tricyclic antidepressants and another showing that yoga may provide benefit as an augmentation strategy for antidepressant treatment. Yoga is commonly used in combination with other treatments for depression; anxiety; and stress-related disorders.

Data on use of yoga for anxiety and depression in older adults are limited. However; one significant study of 69 older adults in India did compare the impact of yoga with Ayurveda or a wait-list control condition on sleep and depressive symptoms $[12,13]$ found that participants in both the yoga and memory enhancement training (MET) groups showed improvements in immediate (Yoga: Cohen's d = 0.95; MET: Cohen's $d=1.06$ ) and delayed recall verbal memory (Yoga: Cohen's $d=0.96$; MET: Cohen's d = 0.59); and immediate (Yoga: Cohen's d = 0.54; MET: Cohen's d $=0.72$ ) and delayed visual memory (Yoga: Cohen's d = 0.48; MET: Cohen's $d=0.64$ ) upon completion of the intervention. Improvements in immediate recall verbal memory (Yoga: Cohen's $\mathrm{d}=0.98$; MET: Cohen's $\mathrm{d}=1.18$ ); delayed recall verbal memory (Yoga: Cohen's d $=0.65$; MET: Cohen's d = 0.34-trend); immediate recall visual memory (Yoga: Cohen's $d=0.39$; MET: Cohen's $d=$ 0.82 ); and delayed recall visual memory (Yoga: Cohen's $d=0.75$; MET: Cohen's $d=0.76$ ) were maintained at the 12 -week followup assessment. However; only yoga participants continued to report improvements in delayed recall verbal memory. Further only the yoga participants demonstrated significantly greater improvement in executive functioning at both post-intervention (Cohen's d ranged from 0.41 to 0.71 ) and 12-week follow-up assessments (Cohen's d ranged from -0.46 to -0.75 and $0.41-0.71$ ). With respect to mood; both groups demonstrated improvements in apathy (Yoga: Cohen's $d=0.52$; MET: Cohen's $d=0.89$ ) but only the yoga group demonstrated improvements in depression (Cohen's $d=-0.62$ ) and resilience (Cohen's $d=0.40$ ) [13].

Numerous studies have identified several molecular pathways targeted by yoga and meditation; including those regulating metabolic and mitochondrial function; neurotransmission and inflammation [14]. In 2014 a novel therapeutic program for treatment of $\mathrm{AD}$; known as metabolic enhancement for neurodegeneration; incorporated yoga and meditation into a regimen targeting multiple aspects of early $\mathrm{AD}$ pathology; achieving sustained cognitive improvement in 9 out 10 patients; including unprecedented quantitative and qualitative improvements after 24 months [14]. These studies provide evidence that yoga or meditative practice contribute to slowing the progression of cognitive decline in AD. However; the molecular mechanisms behind these biological effects have not been properly elucidated [15]. performed a meta-analysis of randomized controlled trials that investigated the effect of physical activity on cognitive function in patients with dementia. They found a positive overall effect of physical activity interventions on cognitive function in patients with dementia in that the effect was driven by interventions that included aerobic exercises and was independent of the type of dementia. Possible physiological mechanisms underlying the positive effects of physical activity interventions on cognitive function in dementia patients have been investigated in experimental animal research; and suggested that physical activity enhances neurotrophin levels; neurogenesis; and vascularization; and mediates neuro-inflammation; and inhibit neuronal integrity [15].

Although multiple studies have assessed the influence of exercise in $\mathrm{AD}$ patients; there is much heterogeneity in the design; outcomes; and exercise intervention used. Interventions range; for example; from individual walking; to group catching and throwing exercises. Outcome variables include physical measures such as walking speed and mobility; as well as changes in cognitive and functional ability [16]. An up-to-date meta-analysis of RCTs concluded that despite variability in intervention procedure; exercise can have a significant effect on reducing global cognitive decline in AD. In this review the authors noted a lack of active or matched control groups in the research; again, limiting the ability to rule out that the effect of exercise could have been produced with other stimulation [16]. Lin and colleagues [17] compared yoga with aerobic exercise in a sample of women with a diagnosis of a psychotic disorder within 5 years of onset. While participants in both conditions experienced improvements in working memory; participants who received yoga also experienced moderate to large improvements in verbal acquisition and attention. Thus; research suggests that yoga may improve attention; processing speed; and verbal memory [17].

At least $66 \%$ of all cases of Alzheimer's disease (AD) are women. Women are two times as likely to develop AD at age 65 as breast cancer and three times as likely at age 75. Khalsa [18] examined the effects of Kirtan Kriya (KK) yoga method on 161 women out of 214 subjects (75\%) across six RCT's with SCD; MCI or stressed family caregivers. Kirtan Kriya is an easy 12 minute/ day yoga meditation. The findings indicate that practicing KK 12 minutes a day for 8-12 weeks improves cognitive function; increases cerebral blood flow; augments neurotransmitters and neuroanatomical plasticity in the anterior and posterior cingulate gyrus; hippocampus; and pre- frontal cortex. Gains were sustained or improved at 6 months [18].

Substantial evidence indicates that physical activity enhances balance; strength; learning; and memory for people of all ages; including those with cognitive impairment such as AD. One activity that may be beneficial to those with cognitive impairment is yoga. High levels of physical activity could maintain cognitive 
function with ageing and reduce the risk of dementia. Possible mechanisms include increased neurogenesis; angiogenesis and synaptic plasticity; mediated by neurotrophic factors (BDNF and IGF-1); Late-life cognitive stimulation could protect against dementia; especially by increasing cognitive reserve

\section{and brain plasticity [17].}

The main purpose of physical exercise for the elderly should be keeping fit; lowering the risk of disability and improving the quality of life. In a program of exercises for elderly people; emphasis should be on increasing flexibility and strengthening muscle. Therefore; practicing hatha yoga can be beneficial. In yoga techniques one adopts a particular body position (asana) slowly; maintains it and relaxes oneself; and then slowly leaves this position. In each asana; one aims at optimal arrangement of the body; elongating the spine by means of active muscle work and correcting one's posture; among other things; due to receptors of deep feeling. The person who practices yoga acquires the skill of maintaining the correct posture; but also expands the range of motion in the joints; improves resistance and muscle strength. Some authors also point hatha yoga as one of the methods of kinesitherapy. Practicing asanas improves the functioning of the whole body; facilitating the work of its individual systems and organs.

Litchke \& Reardon [19] found that a 10-week chair yoga intervention had a significant benefit in slowing the progression of $\mathrm{AD}$ among those with early dementia $(\mathrm{n}=7 ; \mathrm{p}=0.001)$ and that the yoga group had significantly less depression $(n=19 ; \mathrm{p}$ $=0.01$ ). In a similar study; McCaffrey and colleagues [20]; Using a quasi-experimental single-group design; this study examined the feasibility of older adults with Alzheimer's disease (AD)type dementia to complete the Sit 'N' Fit Chair Yoga Program. The findings of this study support the use of the Sit ' $N$ ' Fit Chair Yoga Program as an evidence-based intervention for individuals with moderate to severe AD. All of the older adults with AD who participated in this study completed all 16 yoga sessions and the program held their attention and interest for the full 50 minutes per session. Balance was improved and some improvements in gait speed and walking ability were noted in the data analysis $[19,20]$.

In parallel; several phase 3 trials testing specific Alzheimer's disease treatments were initiated in patients with Alzheimer's disease dementia with the hope of finding a disease-modifying agent able to slow or stop the disease process; but they were unsuccessful; perhaps because intervention at the dementia stage was too late to stop or slow the disease process. Thus; trials testing pharmacological interventions for the prevention of cognitive decline in cognitively normal individuals with positive Alzheimer's disease biomarkers have recently begun. This also provides a window of an opportunity to start yoga therapy early on. There has also been continued interest in lifestyle interventions; with multidomain interventions that target several different lifestyle factors simultaneously attracting much interest [21]. systematic literature review to examine the impact of yoga on persons with mild cognitive impairment (MCI) and dementia indicates that yoga may have beneficial effects on cognitive functioning; particularly on attention and verbal memory. Further; yoga may affect cognitive functioning through improved sleep; mood; and neural connectivity. Thus; providers may consider recommending yoga to persons with MCI or dementia as a safe and potentially beneficial complementary health approach [21-23].

A factor which may influence the effectiveness of exercise is whether the intervention is performed outdoors. Nelson \& Tabet [24] cited the study by Wacker \& Holick in that it has been established that sunlight exposure increases vit D production via absorption of UVB light by 7-hydrocholesterol. Decreased bone mass density has been reported in $\mathrm{AD}$ patients compared to age-matched controls; which was associated with reduced vit D intake and sunlight exposure. Patients with AD assigned to sunlight exposure treatment for one year had a 2.2-fold increase in serum concentrations of vit D and significantly greater bone mass density than a sunlight deprivation group. Physical activity which can be performed outside may provide additional benefits from sunlight exposure [24].

\section{Summary and Discussions}

The practice of yoga can be traced back more than 4000 years and is rooted in the traditions of Vedic culture. A survey of the prevalence and patterns of yoga use in the United States revealed that $63 \%$ of the surveyed yoga practitioners were using the technique for maintenance of wellness and disease prevention; and nearly $48 \%$ were using it for specific health conditions. The surveyors found that $90 \%$ of yoga practitioners felt that it was very or somewhat helpful for their health. In the United States; however; particularly during the last 40 years; yoga has evolved into a more predominantly health oriented rather than spiritually oriented phenomenon [22]. The key difference between yoga and yoga therapy is in the scope of practice; the core competencies of yoga therapists; and their need for clinical training.

Yoga comes from the Sanskrit root for the word yoke. In modern day healthcare this idea of 'yoked' refers to creating health or wholeness in the human experience of the mind; body; and Spirit [22]. In its comprehensive and classic approach; Raja yoga encompasses 8 paths to personal development and well-rounded health. However; Hatha yoga which is more of a fitness-based practice is representative of most yoga practice in western cultures. Hatha yoga consists of 3 basic components including Asana (postures); Pranayama (breath); and Dhyana (meditation) [22,25]. Postures used in Hatha yoga focus on stretching and strengthening muscles as well as relaxation and tension release to improve energy level. Breathing practices used in Hatha yoga are taught to facilitate lengthening; expansion; and stretching of the thorax to promote diaphragmatic breathing and decrease accessory muscle breathing to promote self-awareness 
and decrease sympathetic nervous system control. Meditation practice is designed to bring focus to the current moment to note the state of the body and promote decreased anxiety and depression and increased body awareness [22]. The Indian sage Patañjali prescribed adherence to eight limbs of yoga; aimed at quieting one's mind to achieve the union of mind; body and spirit that is traditional yoga's ultimate goal. These limbs include 'Yama' and 'Niyama' (a code of conduct for an ethical lifestyle); 'Asana' (physical postures); 'Pranayama' (breath control); 'Pratyahara' (withdrawal of the senses from external objects to increase selfawareness); 'Dharana' (concentration); 'Dhyana' (meditation) and 'Samadhi' (oneness with the object of meditation) [22].

Therefore; in addition to working directly with specific medical conditions or injuries; yoga therapists also emphasize raising mindfulness and awareness of both the body and the mind. It has been argued that tension held in the body often originates in the mind and must be dealt with in that realm [26]. The difficulties associated with recommending yoga stem from the low quality of the scientific evidence available regarding its effects. This lack of evidence is partly due to a common failure among researchers to define what they have actually studied. "You soon become aware that [research papers] very often do not define what is meant by yoga"; says Shri Mishra [27]. "There are four traditional schools of yoga Jnana; Bhakti; Karma; and Raja each with subdivisions. They differ greatly in what they demand in terms of physical strength; depth of meditation; breathing control; and spiritual component.

Yet it's very common for reports not to define which type was used in a study. This not only makes it hard to compare results between studies; it also makes it very hard to translate any findings to the bedside" [27]. Research on the medical effects of yoga is continuing. Because yoga may utilize breathing; asanas; visualization; relaxation exercises; and a multitude of other techniques in combination; it may elicit a variety of effects with complexities that are; still not fully understood [25]. "With the limited options for treatment or cure; and the devastation of dementia sequelae; many patients are desperate to find additional options." Patients with cognitive impairment may be additionally susceptible to poor judgment; given their condition; so appropriate counseling about treatment and recommendations becomes critically important [28].

Yoga if not practiced correctly may have adverse effects; this is written in the ancient texts; says Telles [26]. "Research on the mechanisms underlying the effects of yoga also gives insights into what should be avoided or reduced. There are yoga modules for specific diseases based on clinical observations; descriptions in the ancient texts; and rather inconclusive research. Other questions to consider are how much yoga should be practiced (time and intensity) [26]. Patients with physical and mental disabilities may not be well-served in the average commercial yoga studio. Conventional yoga teacher training does not prepare the yoga teacher for patients with specific challenges or medical complexities. Medical yoga therapy is quite different than taking a group yoga class; as it is individualized and prescribed for the patient's particular needs. Even a very qualified yoga instructor may not be knowledgeable; or aware of; an individual student's health issues [29]. Although yoga teacher training certifications include training in anatomy and physiology; this training is not equivalent to the training required by most certified yoga therapists or healthcare practitioners and can be quite varied. Medical Yoga Therapy; prescribed by a physician with special yoga therapy training; offers the most effective; and safest; way to serve the patient with physical or mental disabilities [29]. There are limitations to the use of medical yoga; even when prescribed by a skilled professional. Regular practice of yoga requires energy and commitment; many patients lose this commitment and some (e.g.; depressed patients) may not have the energy for regular practice. In addition; there may be risks to engaging in yoga as well if not prescribed; and practiced; appropriately for the individual [29]. Malthouse \& Fox's findings [30] indicated that a 'one size fits all' approach to developing and delivering activity interventions is not appropriate for people with Alzheimer's disease. Signs of dementia including memory impairment; aphasia; ataxia and behavioral changes may affect participation in physical exercise programs; causing lower attendance and adherence rates; reduced exercise intensity and a higher risk of adverse events [30].

Understanding the specific attitudes; beliefs and values of people with Alzheimer's disease and their care givers may help to motivate and encourage them to engage in activities that are feasible and sustainable [30]. A systematic review by Cramer \& Dobos [31] demonstrated that a considerable proportion of yoga practitioners experienced injuries or other adverse events. However; most of these injuries were mild and transient and risks were comparable to those of non-yoga practitioners. As yoga has been shown to be beneficial for a variety of conditions; it can also be recommended to patients with physical or mental conditions; as long as it is appropriately adapted to their needs and abilities and performed under the supervision of an experienced and medically trained yoga teacher [32].

\section{References}

1. Reitz C, Brayne C, Mayeux R (2011) Epidemiology of Alzheimer disease. Nat Rev Neurol 7: 137-152.

2. Ferrari AJ, Charlson FJ, Norman RE, Patten SB, Freedman G (2013) Burden of depressive disorders by country, sex, age, and year: findings from the global burden of disease study. PLoS Med 10(11): e1001547.

3. Cespon J, Miniussi C, Pellicciari MC (2018) Interventional Programs to Improve Cognition During Healthy and Pathological Ageing: Cortical Modulations and Evidence for Brain Plasticity. Ageing Research Reviews 43: 81-98.

4. Isaacson RS, Ganzer CA, Hristov H, Hackett K, Caesar E (2018) The clinical practice of risk reduction for Alzheimer's disease: A precision medicine approach. Alzheimer's \& Dementia 14(12): 1663-1673.

5. Buchman AS, Boyle PA, Yu L, Shah RC, Wilson RS, et al. (20120 Total daily physical activity and the risk of $\mathrm{AD}$ and cognitive decline in older adults. Neurology 78(17): 1323-1329. 
6. Eyre H, Baune B, Lavertsky H (2015) A Focus on Prevention of Mood and Cognitive Disorders. Psych Clin N Am 38: 495-514.

7. Hewitt J (1993) Yoga. NTC, Publishing Company. Lincoln Wood: Illinois, USA.

8. Kundalini (1996) Yoga Journal World of Yoga. p. 28-30.

9. Stephens I (2017) Medical Yoga Therapy. Children 4(2).

10. Park J, Mc Caffrey R (2012) Chair yoga: Benefits for communitydwelling older adults with osteoarthritis. J Gerontol Nurs 38(5): 12-22.

11. Harvard (2019) Depression and Alzheimer's. News \& Research.

12. Krishnamurthy MN, Telles S (2007) Assessing depression following two ancient Indian interventions: effects of yoga and Ayurveda on older adults in a residential home. J Gerontol Nurs 33(2): 17-23.

13. Eyre HA, Siddarth P, Acevedo B, Van Dyk K, Paholpak P, et al. (2017) A randomized controlled trial of Kundalini yoga in mild cognitive impairment. Int Psychogeriatr 29(4): 557-567.

14. Hassan A, Robinson M, Willerth SM (2020) Determining the mechanism behind yoga's effects on preventing the symptoms of Alzheimer's disease. Neural Regen Res 15(2): 261-262.

15. Groot C, Hooghiestra AM, Raijmakers PGHM, van Berckel BNM, Scheltens P, et al. (2015) The effect of physical activity on cognitive function in patients with dementia: A meta-analysis of randomized control trials. Aging Research Review 25: 13-23.

16. Farina N, Rusted J, Tabet N (2014) The effect of exercise interventions on cognitive outcome in Alzheimer's disease: a systematic review. Int Psychogeriatrics 26(1): 9-18.

17. Lin J, Chan SK, Lee EH (2015) Aerobic exercise and yoga improve neurocognitive function in women with early psychosis. NPJ schizophrenia 1: 15047.

18. Khalsa D S (2018) The Pink Brain Project: How Yoga Meditation May Prevent Alzheimer's in Women. Alzheimer's prevention.org

19. Litchke LG, Hodges JS, Reardon RF (2012) Benefits of chair yoga for persons with mild to severe Alzheimer's disease. Activities, Adaptation \& Aging 36: 317-328.
20. Mc Caffrey R, Park J, Newman D, Hagen D (2014) The Effect of Chair Yoga In older Adults with Moderate and Severe Alzheimer's Disease. Res Gerontol Nurs 7(4): 171-177.

21. Brenes GA, Sohl S, Wells RE, Befus D, Campos CL, et al. (2019) The Effects of Yoga on Patients with Mild Cognitive Impairment and Dementia: A Scoping Review. Am J Geriatr Psychiatry 27(2): 188-197.

22. Cramer H, Lauche R, Dobos G (2014) Characteristics of randomized controlled trials of yoga: a bibliometric analysis. BMC Complement Altern Med 14: 328.

23. Grabara M (2013) Effects of 8-months yoga training on shaping the spine in people over 55. Biomedical Human Kinetics 5: 59-64.

24. Nelson L, Tabet N (2015) Slowing the Progression of Alzheimer's disease; what works? Aging Research Reviews 23: 193-209.

25. Hart J (2008) An Overview of Clinical Applications of Therapeutic Yoga. Alternative \& Complementary Therapies 29-33.

26. Gura ST (2002) Yoga for stress reduction and injury prevention at work. Work 19(1): 3-7.

27. www.thelancet.com

28. Robillard JM (2016) The online environment: a key variable in the ethical response to complementary and alternative medicine for Alzheimer's disease. J Alzheimers Dis 51(1): 11-13.

29. Stephens I (2019) Care report: The Use of Medical Yoga for Adolescent Mental Health. Complementary Therapies in Medicine 43: 60-65.

30. Malthouse R, Fox F (2014) Exploring experiences of physical activity among people with Alzheimer's disease and Their Spouse Carers: a qualitative study. Physiotherapy 100(2): 169-175.

31. Cramer H, Ostermann Th, Dobos G (2018) Injuries and other adverse events associated with yoga practice: A systematic review of epidemiological studies. J Sci Med Sport 21: 147-154

32. Wells RE, Baute V, Wahbeh H (2017) Complementary and Integrative Medicine for Neurologic Conditions. Med clin N Am 101: 881-893.

Your next Submission with Juniper Publishers
will reach you the below assets
- Quality Editorial service
- Swift Peer Review
- Reprints availability
- E-prints Service
- Manuscript Podcast for convenient understanding
- Global attainment for your research
- Manuscript accessibility in different formats
( Pdf, E-pub, Full Text, Audio)
- Unceasing customer service
Track the below URL for one-step submission
https://juniperpublishers.com/online-submission.php

